\title{
A simplified machine learning approach for recognizing human activity
}

\author{
Abdul Lateef Haroon P.S, U. Eranna \\ Department of Electronics and Communication Engineering, Ballari Institute of Technology and Management, India
}

\begin{tabular}{l}
\hline \hline Article Info \\
\hline Article history: \\
Received Jan 31, 2019 \\
Revised Apr 7, 2019 \\
Accepted Apr 19, 2019 \\
\hline
\end{tabular}

\section{Keywords:}

Activity recognition

Classification

Feature extraction

Human activity

Machinelearning

\begin{abstract}
With the wide ranges of real-time event feed capturing devices, there has been significant progress in the area of digital image processing towards activity detection and recognition. Irrespective of the presence of various such devices, they are not adequate to meet dynamic monitoring demands of the visual surveillance system, and their features are highly limited towards complex human activity recognition system. Review of existing system confirms that still there is a large scope of enhancement as they lack applicability to real-life events and also doesn't offer optimal system performance. Therefore, the proposed manuscript presents a model for activity recognition system where the accuracy of recognition operation and system performance are retained with good balance. The study presents a simplified feature extraction process from spatial and temporal traits of the event feeds that is further subjected to the machine learning mechanism for boosting recognition performance.
\end{abstract}

Copyright $($ ) 2019Institute of Advanced Engineering and Science. All rights reserved.

\section{Corresponding Author:}

Abdul Lateef Haroon P.S,

Department of Electronics and Communication Engineering,

Ballari Institute of Technology and Management,

Karnataka, India.

E-Mail: abdulbitm@gmail.com

\section{INTRODUCTION}

With the increasing usage of smart appliances, tracking and monitoring operations have become quite inevitable in almost all the pervasive devices $[1,2]$. One such operation is called a human activity recognition system which is about tracking any specific mobility pattern [3]. A different application of it is already on the commercial markets, e.g. health care, video surveillance, and interaction between human and smart appliance (computing system) [4]. With the upgradation of modern imaging technologies, there is also an improvement in the process of capturing the feed of human-based activities. However, there are various challenges associated with such activity recognition system e.g. variations of intra-classes and similarity of inter-classes, interaction of multiple subjects, background complexity, group activity, etc [5]. There have been various attempts towards usage of machine learning-based approach for solving classification problems in activity-recognition system, e.g. neural network, deep learning, support vector machine, etc [6-10].

However, very less work has considered accuracy as the prominent performance parameters. Apart from this, accuracy in the activity recognition system is not an easy task to be accomplished as various parameters control accuracy, and one prominent parameter in this regard is the potential feature. One of the significant factors that are ignored in the majority of the existing approaches is associated with the identification of the index attribute in the human body that is associated with mobility and displacement. If such index attributes are identified, then it offers enriched information towards feature extraction. The conventional process of capturing human mobility can be carried out on any devices; however, all problems shoot up after the feed is captured owing to the presence of various problems, e.g. illumination, variability, dynamicity of movement, etc. Fig. 1 discusses about different levels of human activity recognition 
system with multiple aspects of applicability on commercial applications (healthcare, recognition of abnormal activity, etc). Theoretically, there are three ways of capturing the human activity for analyzing their behavior in the form of an event. In the bottom level of human activity, they are quite atomic and complex in the form of mobility traits. The second level consists of all sorts of actions or the activities associated with it. The third level is further complicated one as it consists of multiple subjects to be tracked or monitored. The implementation of the proposed system is carried out using various action primitives of the significant limbs of the subjects with different mobility conditions of their limbs. The analysis of the outcome is performed on the basis of such atomic actions focused on specific limb-based actions that consist of various spatial and temporal data arranged in sequence.

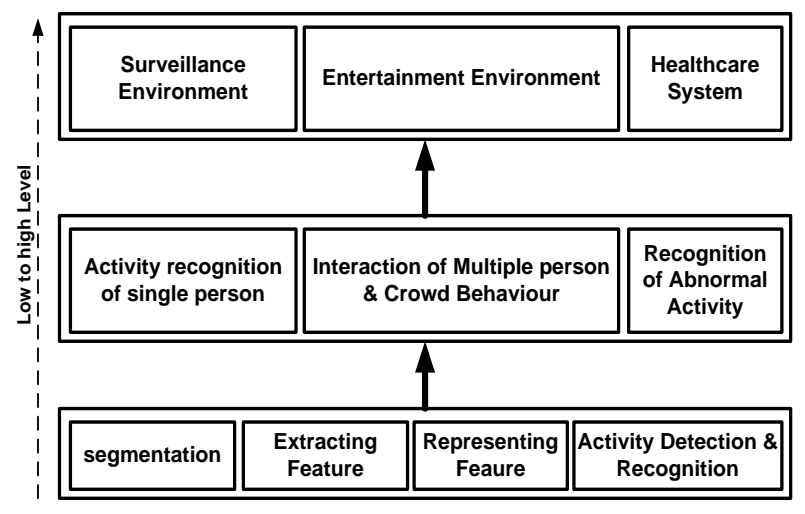

Figure 1. Levels of human activity recognition system

Therefore, the prime objective of the proposed research work is to offer simplified modeling of human activity recognition system where the core emphasis is given to the feature extraction process and accuracy. Existing research-based solutions towards activity recognition are briefed in Section 2 followed by highlighting of the research problem in section 3. Section 4 introduces about the adopted methodology followed by section 5 discussing the algorithm implementation. Discussion of results obtained is carried out in Section 6 while the conclusion is briefed in Section 7.

Our prior review work has offered a brief idea about the research work towards an activity recognition system [11]. The neural network was also adopted by Chen et al. [12] for extracting more information from the event called as knowledge distillation. However, it has been seen that dataset offers importance towards recognition system. Adoption of the sensory dataset is found more frequent (Franco et al. [13]). Plotz and Guan [14] have discussed the significance of deep learning for activity recognition system associated with the mobile computing system. The most recent work of Chen et al. [15] has used convolution neural network considering the distance between joints of inter-frames for performing activity recognition system over the new dataset. There are also good numbers of research where recognition of gesture and activity is captured from Kinect (Gavrilova et al. [16]). The work of Hegde et al. [17] has presented an involuntary mechanism of daily activity recognition using sensory data. Khan et al. [18] have presented a correlational factor as a feature from the activity dataset captured using sensors using body area network.

Khalifa et al. [19] have introduced a unique study where recognition is carried out from energy harvesting data. Similar line of research is also carried out by Hsu et al. [20] where principal component analysis is used for dimensional reduction Usage of Hidden Markov Model has been used for constructing a framework for activity recognition as seen in work of $\mathrm{Li}$ et al. [21] along with usage of k-means clustering for feature extraction. Hidden Markov model and supervised classification technique are also reported to be used in the activity recognition system (Sok et al. [22]). Recognition system over online data has been reported by Qi et al. [23] considering the dictionary learning mechanism. It was also reported in the existing system that in-depth learning-based training approach could be used for image-based activity recognition system (Wang et al. [24] and Tan et al. [25]). Usage of the network, e.g., WiFi signal and radio-frequencies were also reported to be used in human activity recognition Wang et al. [26], Wenyuan et al. [27] Activity recognition on the basis of smart-based data are discussed by Wang et al. [28]. Other unique research implementations are also witnessed in work of Yao et al. [29] Yang et al. [30] and a unique recurrent modeling is presented using dilation operation for activity recognition system (Xi et al. [31]). The next section discusses the research problem. 
After reviewing the existing mechanism associated with an activity recognition system, it is found that there is very less work being carried out towards addressing the conventional challenges associated with it. The approaches are not reported to mitigate the problems of variation of within classes, clutter existing in the background, occlusion, and condition of illumination and poses of the subject (human). A closer look into the existing approaches will show that the majority of the existing research approaches towards activity recognition system is carried out over sensory data and not much on images. Although, this mechanism offers simplified approaches they were never accessed for their accuracy rate. Apart from this, the test environment is only restricted to the static dataset of activity recognition, and no much analysis has been carried out for online dynamic real-time feeds of events. However, such approaches are never able even to identify the problematic fact that amalgamation of various action-based features is never sufficient to obtain the variations associated with the images as well as diverse attributes connected to it, e.g., motion, shape, appearance, etc.

The usage of the neural network is too much abundant and not much flexibility is offered towards classification problems associated with the human activities. Therefore, the classifying the real-time feed is one challenging aspect in activity recognition. In order to incorporate a better form of accuracy, it is required that the framework of the activity recognition system should carry out an effective extraction of the feature, precise representation of feature followed by classification of the pattern. The proposed work captures the real-time feed of the subject in order to obtain the depth information. However, such form of real-time captures will be associated with noises too that can adversely affect the precision of recognition. Therefore, the initial step includes the elimination of all forms of outliers as well as missing data. The proposed work is an extension of our prior work [32] towards an activity recognition system where the current work completely emphasizes improving the accuracy of the identified activity. The proposed system identifies all the activities on the basis of the limb movement of the human body, and hence better accuracy can be maintained. Therefore, the proposed system hypothesizes that if the significant limb movements can be identified and recognized that any form of application-oriented human activity can be developed commercially.

Figure 2 highlights the mechanism of taking the event feed as an input which undergoes an extraction process of its index points of mobility along with depth map. The proposed system makes use of sequential relative joint angle existing between two varied skeletal points in order to construct a matrix for retaining a specific mobility pattern of the subject. This information of specific subject further assists in recognition of the activity once the matrix is subjected for training. All the significant mobility points associated with the subject is retained in a matrix from where temporal and spatial information has been used for extracting features. The extracted features are then subjected to the machine learning mechanism in order to perform activity classification. Adoption of machine learning mechanism assists in the selection of most relevant sequences of a relative joint angle obtained from training as well as testing data sample for classification of the activity. The next section will discuss the algorithm implemented for this purpose.

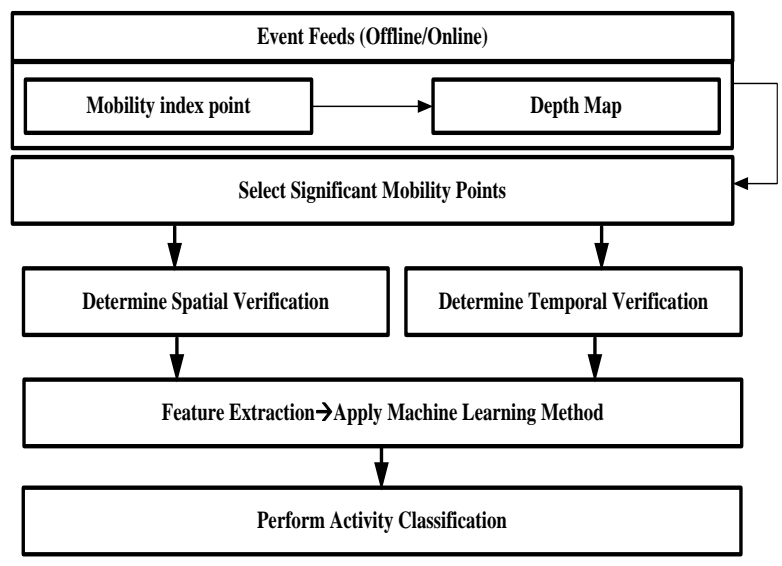

Figure 2. Schematic architecture of proposed system

\section{ALGORITHM IMPLEMENTATION}

The development of the proposed algorithm initiates with the representation of the human activity, and therefore the first step is to construct a matrix for extraction potential features. This will enable the retention of maximum accuracy as well as optimize the space for computation. The proposed system makes 
use of two forms of feature for directly representing the significant activity viz. spatial-based features $\rho_{1}$ and temporal-based features $\rho_{2}$. Using the framework of our prior work [32], the normalization of the joint coordinates is carried out from the real-time feeds of the subjects (human) for capturing the events. The referential point of the coordinate system can be obtained by averaging the three-dimensional coordinates corresponding to center, left, and right side of the subject. The first algorithm is responsible for recording the event as follows:

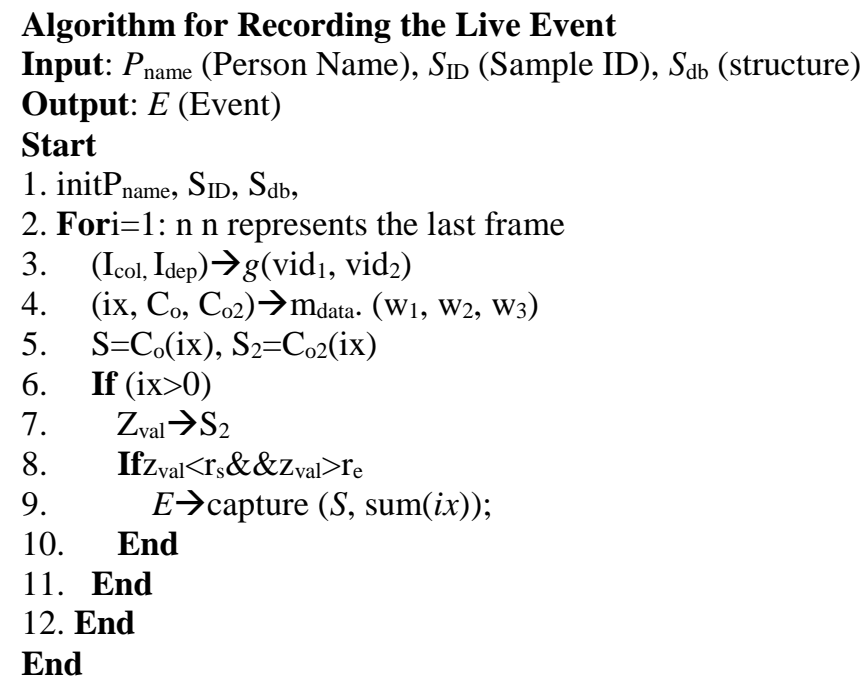

This algorithm is responsible for capturing the live events that have a series of human activity. In order to narrow down the analysis, the proposed system performs a selection of a human subject and represents them with respect to two variables viz. $P_{\text {name }}$ (Person name) and $S_{\mathrm{ID}}$ (Sample ID) (Line-1). The real-time feed of the event is captured with respect to the color image and depth image that are represented as variables vid ${ }_{1}$ and $\mathrm{vid}_{2}$ respectively (Line-3). A function $g(\mathrm{x})$ is created to take the feed of both the real-time events of the human subject and store it in two discrete matrices of image color $I_{\text {col }}$ and image depth $I_{\text {dep }}$ (Line-3). In order to offer more granularity in the approach, the object associated with vid ${ }_{1}$ is stored in Icol, but the object associated with $v_{2}$ is stored in three different matrices, i.e., image depth, metadata depth, and temporal-spatial depth. A tracker variable $i x$ is constructed that is responsible for tracing $S_{\mathrm{ID}}$ (Line-4) while a matric $C_{\mathrm{o}}$ and $C_{\mathrm{o} 2}$ are developed for storing the two- and three-dimensional coordinates respectively. Therefore, the variable $S$ and $S_{2}$ is a representation of the processed matrix of tracker $i x$ (Line-5). In case there are positive numbers of the tracker/indexer of the events (Line-6), then a new matrix $\mathrm{Z}_{\mathrm{val}}$ is constructed for capturing all the event related information in the form of $\mathrm{S}$ and sum of all the tracker variable ix (Line-9).

The algorithm of the proposed system is designed for precise identification of the human activity with the aid of a simple machine learning mechanism. The idea of using a machine learning mechanism is targeted for offering accuracy in the identification process. In order to incorporate a greater degree of accuracy in the process of the activity recognition system, it is necessary that every smaller piece of information associated with the event should be recognized. Therefore, the proposed system implements a mechanism where the features are built up from spatial and temporal attributes connected to the real-time events in the proposed system. The algorithm takes the input of real-time event video in the form of $\mathrm{I}_{\mathrm{s}}$ (input sample) which after processing yields an outcome of $s_{\text {feat }}$ (sampled feature). The step involved in the proposed algorithm is as follows:

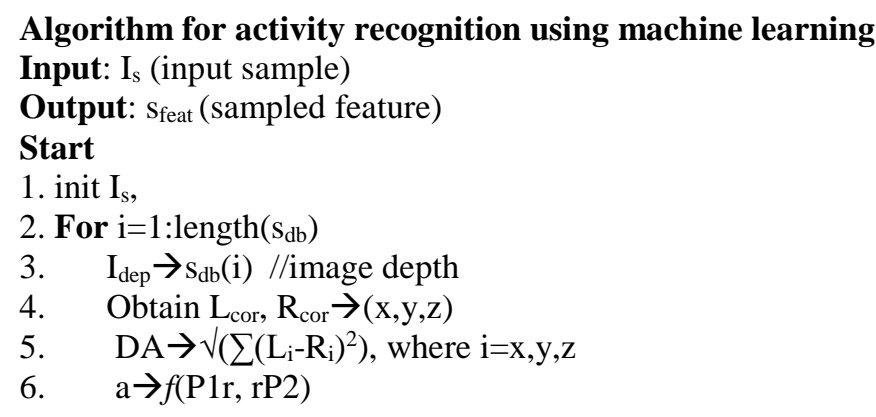




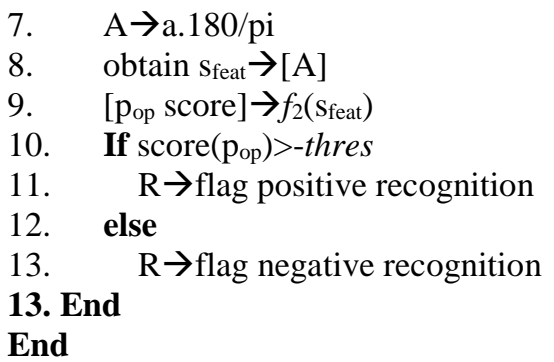

Algorithm Step Discussion: For simpler processing of real-time feed of an event, the proposed system digitizes the feed in order to obtain it as $I_{s}$ (input sample) which has both spatial and temporal information (Line-1). The next part of the algorithm is to obtain significant features from all the spatial data $\left(\mathrm{s}_{\mathrm{db}}\right)$, which is further used for extracting the image depth $\mathrm{I}_{\mathrm{dep}}$ (Line-3). In this stage, both the colored version of the core skeleton as well as image depth is obtained. The colored image $S$ is obtained from the data structure of $s_{\mathrm{db}}$ while image depth $\mathrm{I}_{\mathrm{dep}}$ is obtained from Line-3. The next part of the algorithm is focused on obtaining various potential features associated with the ankle and wrist as they are a prominent indicator of any significant activity. The proposed algorithm obtains the three-dimensional coordinates system from both the left and right ankle (Line-4). A Euclidean distance is applied for computing spatial the differences between the left and right ankle (Line-5) considering all the three-dimensional coordinates. Similar algorithmic steps, i.e. Line-4and Line-5 is used for computing the Euclidean distance between the left and the right wrist too.

Although, the logic focuses on the identifying the spatial attribute of the displacement considering the significant limbs, e.g., ankle and wrist, however, the temporal attribute of the displacement can be known if the orientation measure is considered for the event captured.Therefore, the algorithm performs the computation of the orientation measurement of another limb, i.e. both the elbow. The algorithm computes the effective displacement of both the elbows viz. P1r and $\mathrm{rP} 2$ and applies a function $f(\mathrm{x})$ on this to obtain the actual orientation factor associated with the elbow (Line-). The function $f(\mathrm{x})$ computes inverse tangent of four quadrants over two variable viz. i) variable-1 for normalized cross product of $\mathrm{P} 1 \mathrm{r}$ and $\mathrm{rP} 2$ and ii) dot product of P1r and rP2. Finally, the orientation score is obtained A (Line-7). This mechanism is performed for both elbow and legs orientation to finally obtain all the features sfeat from A (Line-8). The algorithm applies another function $f_{2}(\mathrm{x})$ over the obtained features to extract the final set of features (Line-9). However, the obtained feature is just for feature identification that directly assists in the activity recognition system. The operation carried out till this stage ensure $70 \%$ of accuracy owing to the adoption of spatial and temporal based features; therefore, the proposed system upgrade the function $f_{2}(\mathrm{x})$ using machine learning based approach.

There are various justifications of using machine-based learning approach. Adoption of this approach allows the implementation of multiple forms of machine learning approaches for the purpose of efficient predictive recognition of human activity. The proposed system performs prediction of data with respect to the data history, and hence all the factors (spatial as well as temporal aspects) are considered while performing the training operation. When this function is applied on a set of features, the training yields predictive operator $\mathrm{p}_{\mathrm{op}}$ and its respective score (Line-9). After obtaining the value of the score, it is compared with the cut-off value thres where the positive value will ensure perfect recognition $\mathrm{R}$ of the activity (Line-11) of the event while the negative value will call for negative recognition $\mathrm{R}$ (Line-13). A closer look into this step of operation will show that this is meant for improving the accuracy of the recognition capability. Although the process is bit iterative in this regards, interestingly it offers the capability to reduce down the error-prone identifications. According to the algorithm, if the process does find a perfect match with its trained dataset with respect to the spatial and temporal factor, it will mean that the subject displays a very different movement and hence the recognition system captures that the subject is different and distinct. Therefore, the proposed system offers an extensive granularity in its outcome for activity recognition system that enables the system to perform a good balance between accuracy as well as identification performance.

\section{RESULT ANALYSIS}

This section discusses the outcomes obtained from the implementation of the proposed algorithm towards the identification of the human activity. The prime strategy to analyze the outcome is to assess the mobility of the subject in order to determine their precise activity identification. The study considers approximately 20 skeletal joints. Using the similar test environment of our prior experiment [32], the proposed system has captured a $20 \times 20$ matrix associated with all the sequences of the individual video. 
In this system, all the cells in the matrix depict the sectors that are occupied by histograms. Different numbers of subjects are considered for this experiment where different video sequences have been captured and stored. The scripting of the proposed logic was carried out in MATLAB, and it offers supportability of both life as well as offline analysis.

Figure 3 highlights the visual outcomes of the proposed system where it can be seen that real-time feeds are captured from the visual sensors in order to obtain the colored image as well as depth image. This information will assist in extraction spatial and temporal-based features that will finally assist in improving the accuracy of the proposed activity recognition system. The analysis has been carried out using 35 subjects (only four different sample test subjects are shown in Figure 2). The next part of the analysis is all about assessing accuracy. After the capturing of the event has been carried out, the proposed system computes allthe essential mobility aspects associated with the ankle, elbow, wrist, leg, etc. Figure 4 highlights the computation of the ankle and wrist displacement. Figure 5 and Figure 6 shows that the proposed system is capable of tracking all the minor and insignificant movement of the subject too. The system also tracks information associated with ankle, wrist, elbows, and leg as shown inFigure 5. The proposed system also estimates the mean matrix as well as stream map as exhibited in Figure 7 that offers the comprehensive evidence of accuracy obtained for all the activity identification.

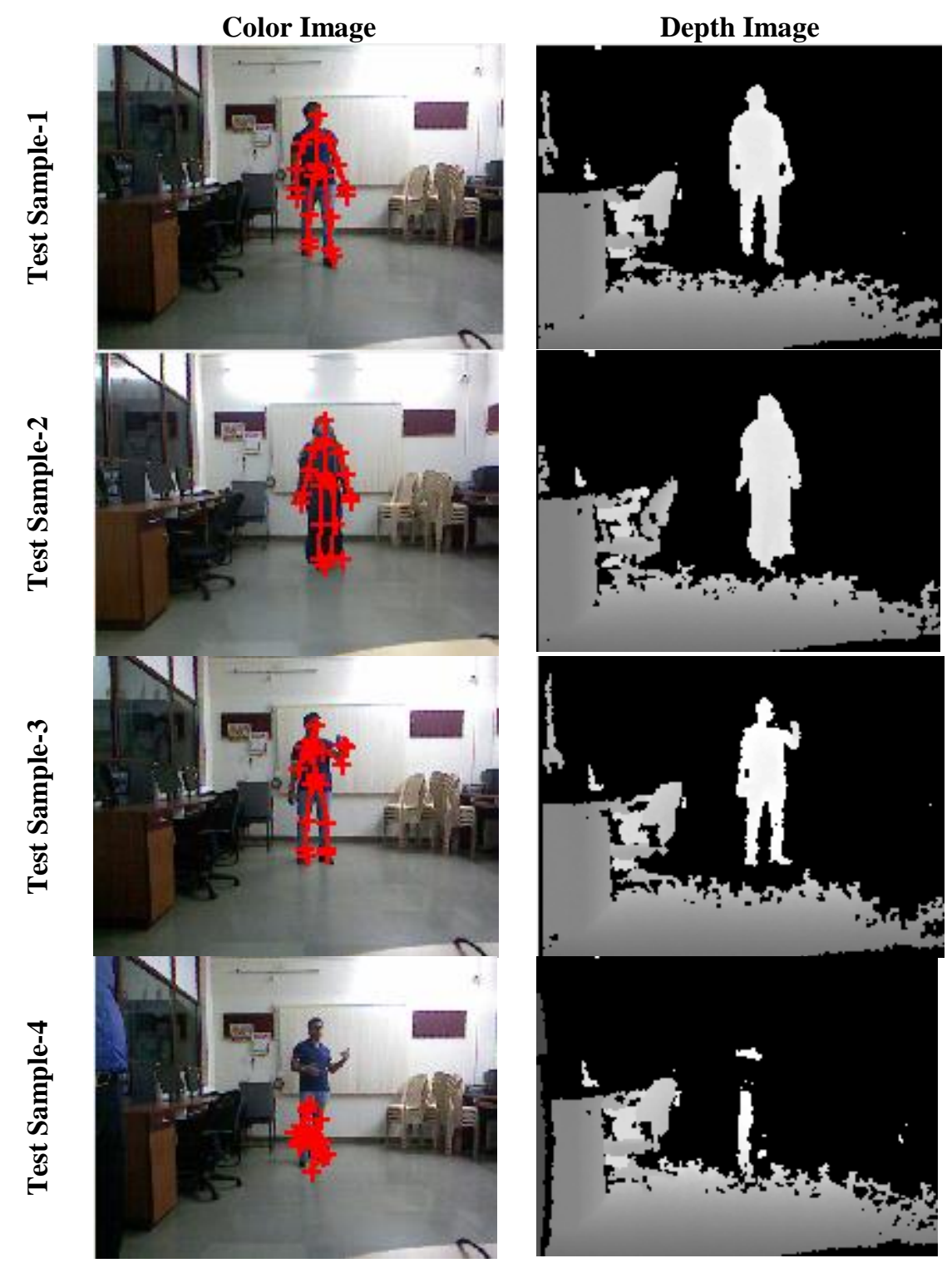

Figure 3. Visual outcomes of proposed activity recognition 


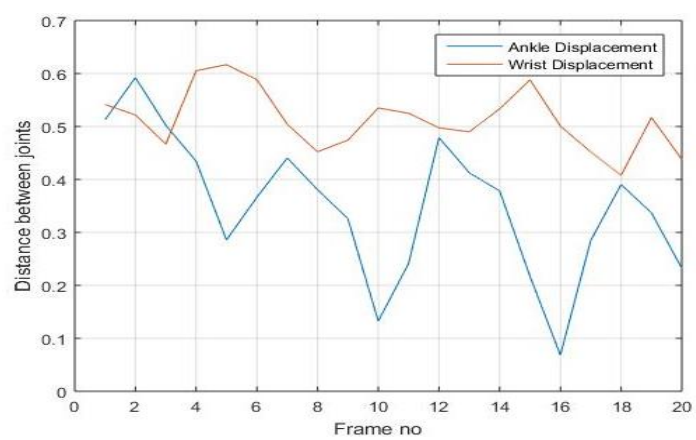

Figure 4. Wrist and ankle displacement calculation

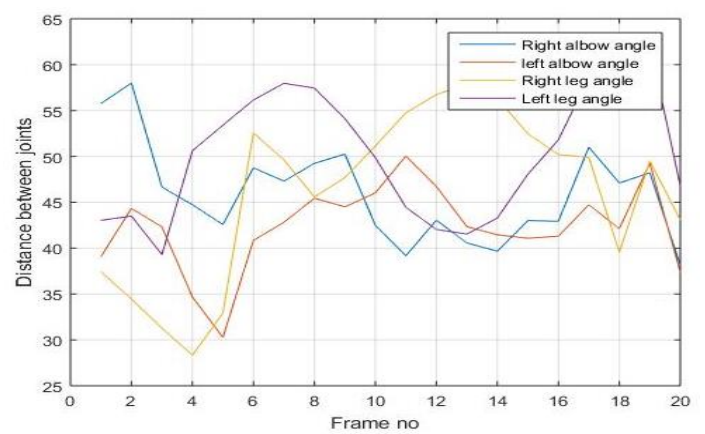

Figure 5. Leg and elbow calculation
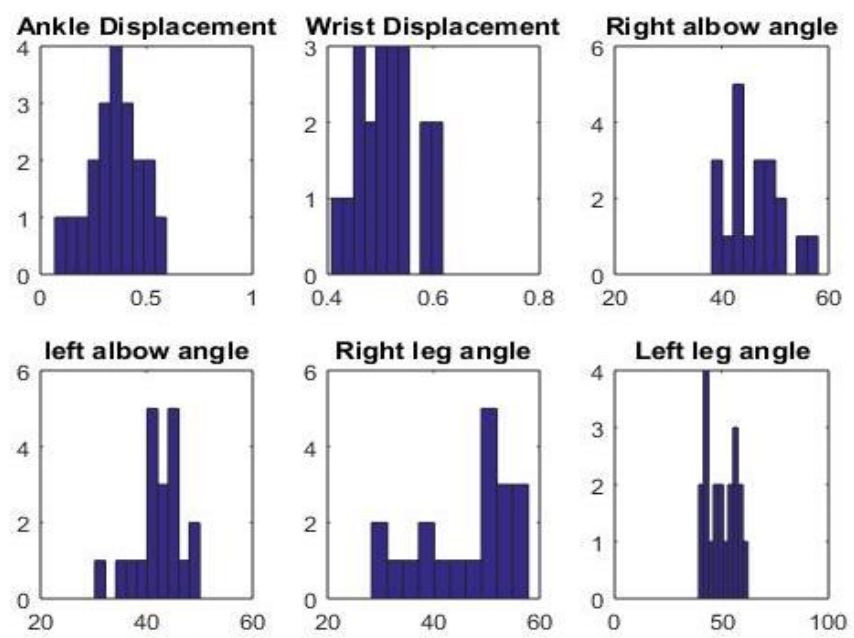

Figure 6. Event Attribute Detection using Histogram

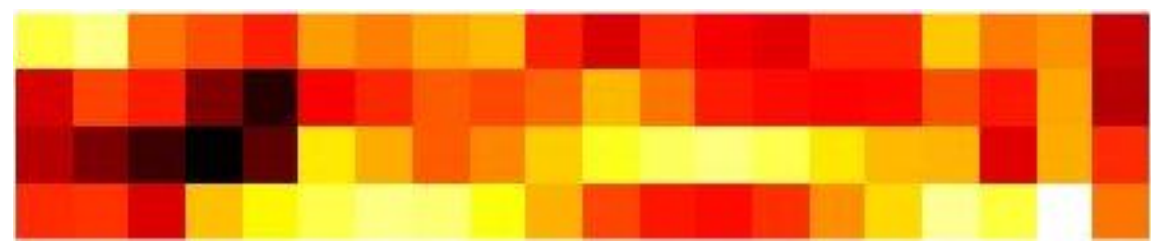

Figure 7. Matrix representations for different histograms

The idea of the proposed system is assessed with respect to the existing approaches of activity recognition system, e.g. Zdraveski et al. [33], Nakagawa et al. [34], and Henpraserttae et al. [35]. The summarized outcome is highlighted in Figure1.

The comparative analysis is shown in Figure 8 highlights that the proposed system offers better accuracy as compared to existing approaches considered for analysis. Although, the approach of Zdraveski et al. [33] is found to be $95 \%$, still, its practical applicability, as well as computational complexity, is not computed. The approach of Nakagawa et al. [34] suffers from higher dependencies of massive data for identification. Similarly, the work of Henpraserttae et al. [35] doesn't consider the problem of data variability, and hence they are applicable only for static datasets without any assessment of its computational complexity. The proposed system has exhibited $10-15 \%$ of memory consumption with 0.5341 seconds of processing time consumption over $532 \mathrm{MB}$ of digitized data of the real-time event. Hence, the supportability of the proposed system is quite higher on practical ground. It should be noted that all the existing system are experimented and analyzed on the basis of the standard dataset. Although, it is least important about the format of the data, however, analysis over static dataset doesn't prove the practicality of the existing solution. Moreover, the existing concepts towards activity recognition are quite hypothetical as they were 
never assessed for their computational complexity. However, the proposed system bridges this gap and proves that it maintains a good balance between accuracy and its other associated performance. The performance of the result is the same for both real-time captures of subject mobility as well as static frames too.

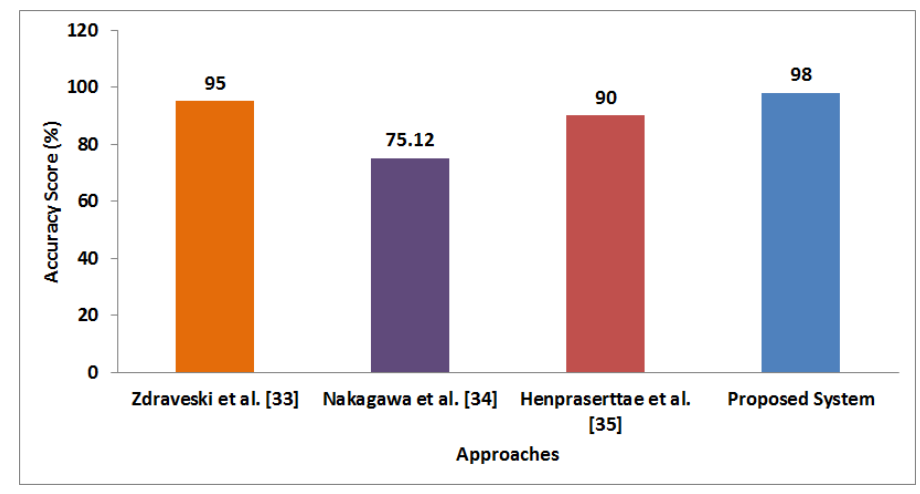

Figure 8. Comparative analysis of accuracy score $(\%)$

\section{CONCLUSION}

Recognition of the specific activity of the human is quite a difficult task as it suffers from various challenges associated with the dynamicity of movement as well as variation in the illumination condition. The outcome exhibits that features captured on the basis of spatial as well as temporal factors are highly suitable for assessing all the significant mobility/displacement of the subjects. The recognition of the activity was captured in the form of displacement of various significant limbs of the subject as seen from histogram analysis. The study outcome shows that machine-based learning approach is highly fruitful in obtaining better results in the form of accuracy as well as overall system performance. Therefore, the technical viability, as well as the applicability of the proposed system, is quite high in a practical environment.

\section{REFERENCES}

[1] M.H. Hugos, "Essentials of Supply Chain Management," John Wiley \& Sons, Business \& Economics, pp. 368, 2018.

[2] F.D. 1. Prieta, et al., "Trends in Cyber-Physical Multi-Agent Systems,"The PAAMS Collection - 15th International Conference, PAAMS 2017, Springer, pp. 340, 2017.

[3] C. Antoniou, et al., "Mobility Patterns, Big Data and Transport Analytics: Tools and Applications for Modeling,"Elsevier, pp. 452, 2018.

[4] W. Zhao, et al., "Recent Developments in Smart Healthcare,"Computers, pp. 362, 2018.

[5] A. E. Hassanien, et al., "Advanced Machine Learning Technologies and Applications,"First International Conference, AMLTA 2012, Cairo, Egypt, December 8-10, 2012, Proceedings, Computers, pp. 602, 2012.

[6] M. A. Labrador and O. D. L. Yejas, "Human Activity Recognition: Using Wearable Sensors and Smartphones," CRC Press, pp. 207, 2013.

[7] Y. Ma and G. Gua, "Support Vector Machines Applications," Springer Science \& Business Media, pp. 302, 2014.

[8] T. Wuest, "Identifying Product and Process State Drivers in Manufacturing Systems Using Supervised Machine Learning,"Springer, pp. 272, 2015.

[9] E.Banguero, et al., "Renewable microgrid operational results and economic evaluation using RETScreenTM,"International Journal of Electrical and Computer Engineering (IJECE), vol/issue: 9(2), pp. 723$731,2019$.

[10] K. Dhineshkumar, et al., "Performance analysis of PV powered multilevel inverter,"International Journal of Electrical and Computer Engineering (IJECE), vol/issue: 9(2), pp. 753-760, 2019.

[11] A. L. Haroon P. S. and U.Eranna,"Insights on Research-based Approaches in Human Activity Recognition System,"Communications on Applied Electronics, vol/issue: 7(16) pp. 23-31, May 2018.

[12] Z. Chen, et al., "Distilling the Knowledge from Handcrafted Features for Human Activity Recognition,"in IEEE Transactions on Industrial Informatics, vol/issue: 14(10), pp. 4334-4342, Oct 2018.

[13] E. De-La-Hoz-Franco, et al., "Sensor-Based Datasets for Human Activity Recognition - A Systematic Review of Literature,"in IEEE Access, vol. 6, pp. 59192-59210, 2018.

[14] T. Plötz and Y. Guan, "Deep Learning for Human Activity Recognition in Mobile Computing,"in Computer, vol/issue: 51(5), pp. 50-59, May 2018. 
[15] Y. Chen, et al., "Robust Activity Recognition for Aging Society,"in IEEE Journal of Biomedical and Health Informatics, vol/issue: 22(6), pp. 1754-1764, Nov 2018.

[16] M. L. Gavrilova, et al., "Kinect Sensor Gesture and Activity Recognition: New Applications for Consumer Cognitive Systems,"in IEEE Consumer Electronics Magazine, vol/issue: 7(1), pp. 88-94, Jan 2018.

[17] N. Hegde, et al., "Automatic Recognition of Activities of Daily Living Utilizing Insole-Based and Wrist-Worn Wearable Sensors," in IEEE Journal of Biomedical and Health Informatics, vol/issue: 22(4), pp. 979-988, Jul 2018.

[18] M. U. S. Khan, et al., "On the Correlation of Sensor Location and Human Activity Recognition in Body Area Networks (BANs),"in IEEE Systems Journal, vol/issue: 12(1), pp. 82-91, Mar 2018.

[19] S. Khalifa, et al., "HARKE: Human Activity Recognition from Kinetic Energy Harvesting Data in Wearable Devices,"in IEEE Transactions on Mobile Computing, vol/issue:17(6), pp. 1353-1368, Jun 2018.

[20] Y. Hsu, et al., "Human Daily and Sport Activity Recognition Using a Wearable Inertial Sensor Network,"in IEEE Access, vol. 6, pp. 31715-31728, 2018.

[21] W. Li, et al., "Log-Likelihood Clustering-Enabled Passive RF Sensing for Residential Activity Recognition,"in IEEE Sensors Journal, vol/issue: 18(13), pp. 5413-5421, Jul 2018.

[22] P. Sok, et al., "Activity Recognition for Incomplete Spinal Cord Injury Subjects Using Hidden Markov Models,"in IEEE Sensors Journal, vol/issue: 18(15), pp. 6369-6374, Aug 2018.

[23] J. Qi, et al., "Learning Complex Spatio-Temporal Configurations of Body Joints for Online Activity Recognition,"in IEEE Transactions on Human-Machine Systems, vol/issue:48(6), pp. 637-647, Dec 2018.

[24] J. Wang, et al., "Device-Free Wireless Localization and Activity Recognition: A Deep Learning Approach,"in IEEE Transactions on Vehicular Technology, vol/issue: 66(7), pp. 6258-6267, Jul 2017.

[25] T. Tan, et al., "Multi-Resident Activity Recognition in a Smart Home Using RGB Activity Image and DCNN,"in IEEE Sensors Journal, vol/issue: 18(23), pp. 9718-9727, Dec 2018.

[26] C. Wang, et al., "Literature review on wireless sensing-Wi-Fi signal-based recognition of human activities,"in Tsinghua Science and Technology, vol/issue: 23(2), pp. 203-222, Apr 2018.

[27] L. Wenyuan, et al., "From Lens to Prism: Device-Free Modeling and Recognition of Multi-Part Activities,"in IEEE Access, vol. 6, pp. 36271-36282, 2018.

[28] L. Wenyuan, et al., "From Lens to Prism: Device-Free Modeling and Recognition of Multi-Part Activities,"in IEEE Access, vol. 6, pp. 36271-36282, 2018.

[29] L. Yao, et al., "Compressive Representation for Device-Free Activity Recognition with Passive RFID Signal Strength,"in IEEE Transactions on Mobile Computing, vol/issue: 17(2), pp. 293-306, Feb 2018.

[30] Z. Yang, et al., "DFTerNet: Towards 2-bit Dynamic Fusion Networks for Accurate Human Activity Recognition,"in IEEE Access, vol. 6, pp. 56750-56764, 2018.

[31] R. Xi, et al., "Deep Dilation on Multimodality Time Series for Human Activity Recognition,"in IEEE Access, vol. 6, pp. 53381-53396, 2018.

[32] A. L. Haroon P.S. and U. Eranna, "An Efficient Activity Detection System based on Skeleton Joints Identification,"International Journal of Electrical and Computer Engineering (IJECE), vol/issue: 8(6), pp. 4995-5003, 2018.

[33] A. Henpraserttae, S. Thiemjarus and S. Marukatat, "Accurate Activity Recognition Using a Mobile Phone Regardless of Device Orientation and Location," 2011 International Conference on Body Sensor Networks, Dallas, TX, 2011, pp. 41-46

[34] E. Nakagawa, K. Moriya, H. Suwa, M. Fujimoto, Y. Arakawa and K. Yasumoto, "Toward real-time in-home activity recognition using indoor positioning sensor and power meters," 2017 IEEE International Conference on Pervasive Computing and Communications Workshops (PerCom Workshops), Kona, HI, 2017, pp. 539-544.

[35] E. Zdravevski et al., "Improving Activity Recognition Accuracy in Ambient-Assisted Living Systems by Automated Feature Engineering," in IEEE Access, vol. 5, pp. 5262-5280, 2017.

\section{BIOGRAPHIES OF AUTHORS}

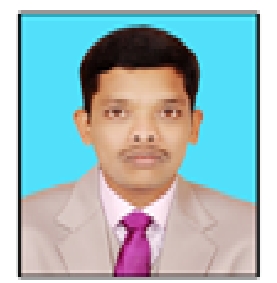

Abdul Lateef Haroon P.S., currently working as an Assistant Professor in the Department of Electronics and Communication Engineering, Ballari Institute of Technology and Management. I have completed B.E from RYMEC College, Ballari during 2008-2012 with aggregate 72\%; I have done M. Tech from KSSEM College, Bangalore during 2012-2014 with aggregate 84\%. I am Currently Pursuing Ph.D. from VTU with BITM College as a Research centre. I have published 7 IEEE Conference Papers and 20 International Journals. My areas of Interest are Digital Signal Processing, Digital Image Processing, Computer Vision, ARM Microcontrollers, and Neural Networks.

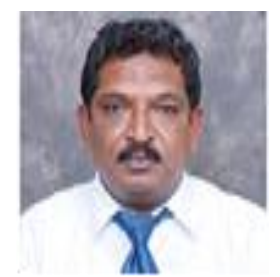

U. Eranna, Currently working as Professor and HOD in the Department of Electronics and Communication Engineering, Ballari Institute of Technology and Management. He has published 8 conference papers and 20 journal papers. His area of research was Communication, Control System 